\title{
Bounds of imaginary spectra of LTI systems in the domain of two of the multiple time delays QINGBIN GAO $^{\text {a }}$, NEJAT OLGAC ${ }^{* b}$
}

\author{
${ }^{a}$ Department of Mechanical and Aerospace Engineering, California State University Long Beach, Long Beach, CA 90840, United States; \\ E-mail address: Qingbin.Gao@csulb.edu \\ * Corresponding author. Phone +1 860 4862382; Fax +1 860 4865088; \\ ${ }^{\mathrm{b}}$ Mechanical Engineering Department, University of Connecticut, Storrs, CT 06269, United State; E-mail address: olgac@engr.uconn.edu
}

\begin{abstract}
The stability of linear time invariant (LTI) systems with independent multiple time delays and the cluster treatment of characteristic roots (CTCR) paradigm are investigated from a new perspective. It is known that for such systems, all the imaginary characteristic roots can be detected completely on a small set of hypersurfaces in the domain of the delays [34]. They are called kernel hypersurfaces $(\mathrm{KH})$. The complete description of $\mathrm{KH}$ is the only prerequisite for the CTCR stability assessment procedure. As the number of delays increases, however, their evaluation becomes infeasible. Instead, we present a procedure to extract the 2-D cross-sections of these hypersurfaces in the domain of any two of the delays by fixing the remaining delays. In the 2-delay domain of interest, the exact upper and lower bounds of the imaginary spectra are determined. For this, a combination of half-angle tangent representation of the characteristic equation and the Dixon resultant theory is used as the main contributions of this paper. The complete $\mathrm{KH}$ are obtained by sweeping the root crossing frequency in this interval. Using this knowledge CTCR creates the cross-section of the stability map in the domain of the two arbitrarily selected delays. We demonstrate the effectiveness of this methodology over an example case study with three independent delays and two commensurate ones.
\end{abstract}

Key words: Time-delay systems, multiple delays, stability, CTCR, kernel hypersurfaces.

\section{Introduction}

This article concerns the asymptotic stability analysis of a general retarded class of linear time-invariant multiple time delay systems (LTI-MTDS):

$$
\dot{\mathbf{x}}(t)=\mathbf{A} \mathbf{x}(t)+\sum_{k=1}^{p} \mathbf{B}_{k} \mathbf{x}\left(t-\tau_{k}\right)
$$

where $\mathbf{x} \in \mathfrak{R}^{n}$ is the state vector; $\mathbf{A}, \mathbf{B}_{k}, k=1, \ldots, p$ are constant and known matrices in $\mathfrak{R}^{n \times n}$ and $p$ is the number of independent delays in the system. Boldface capital notation is used for vector and matrix quantities. The characteristic equation of this system is

$$
g(s, \boldsymbol{\tau})=\operatorname{det}\left(s \mathbf{I}-\mathbf{A}-\sum_{k=1}^{p} \mathbf{B}_{k} e^{-\tau_{k} s}\right)=0
$$

where $\boldsymbol{\tau}=\left(\tau_{1}, \tau_{2}, \ldots, \tau_{p}\right) \in \mathfrak{R}^{p+}$ is the delay vector. The delays, at the most stringent case, are assumed rationally independent from each other.

The analysis of asymptotic stability of systems in (2) within the domain of the delays is a fundamental problem in the controls community ([11], [12], [12], [14], [17], [19], [27]). It is also known to be an N-P hard class mathematical problem [38]. This task becomes much more complex as the number of delays, $p$, increases [22]. For $p \geq 3$, only a limited number of reports exist in the literature ([1], [16], [32], [33], [36]). First three deal with a similar subclass of MTDS excluding commensurate and cross-talking delay terms in the corresponding characteristic equation. The method in [33] treats the most general MTDS (1) for determining some critical features but fails to declare that this process is only a preparatory phase to an umbrella stability paradigm, and as such it does not stand by itself as a stability method.

In this paper, we deploy the Cluster Treatment of Characteristic Roots (CTCR) paradigm for the task [34]. CTCR requires the complete knowledge of the loci in the delay space on which the system exhibits purely imaginary characteristic roots. These loci are composed of two sets: kernel and offspring hypersurfaces $(\mathrm{KOH})([34],[10])$. The kernel hypersurfaces $(\mathrm{KH})$ consist of points that exhibit the smallest positive delay values on all of the $p$ delays. Entire offspring hypersurfaces $(\mathrm{OH})$ are obtained from $\mathrm{KH}$ by a pointwise nonlinear transformation [34] as will be explained later. That is, the mere knowledge of $\mathrm{KH}$ is sufficient to obtain the complete infinite set of $\mathrm{OH}$. A number of mathematical procedures are available in the literature to determine the $\mathrm{KOH}$, such as Rekasius substitution [31], Kronecker summation [9], and matrix pencil [28]. For MTDS with $p>3$. However, the calculation of the $\mathrm{KOH}$ in the $p$-dimensional $(p-\mathrm{D})$ delay space is known to be computationally infeasible [22]. Instead, we fix all but two of the delays arbitrarily and examine the intersections of $\mathrm{KOH}$ on the domain of the 2 delays. For this, a frequency sweeping technique is deployed, that is completely numerical [6]. It is recognized 
to be more effective than those techniques that require symbolic computations especially for systems with higher orders and higher number of delays [30] . For the frequenfrequency sweeping technique, however, precise knowledge of the upper and lower bounds of the imaginary spectra are needed. These bounds are known to exist for the retarded time delay systems (1) [18]. The main contribution of this paper is in their determination. To achieve this we deploy a combination of half-angle tangent substitution method [37] and the Dixon resultant theory ([3], [7], [24]) for the first time in literature.

The paper is structured as follows: Section 2 reviews some key definitions of MTDS and half-angle tangent method. The main results on determining the bounds of the imaginary spectra and the 2-D cross-section of the stability map for MTDS with more than three delays are given in Section 3. Section 4 demonstrates the strength of the proposed methodology over an example case study with three independent and two commensurate delays $(p=5)$.

\section{Preliminaries}

An LTI-MTDS (1) is asymptotically stable if and only if all its infinitely many characteristic roots are on the left half of the complex plane. The continuity of these roots with respect to delays has already been established in the literature ([18], [20]). Since the kernel and offspring hypersurfaces $(\mathrm{KOH})$ are the only loci where the characteristic equation (2) possesses imaginary roots, they are the only potential stability switching locations (thus often referred to as "stability switching hypersurfaces"). For a complete stability map one needs to determine all of these $\mathrm{KOH}$ exhaustively. As stated in Section 1, the mere knowledge of $\mathrm{KH}$ is sufficient for this. The determination of $\mathrm{KH}$ in $p$-D delay space is, however, computationally infeasible for $p>3$. Instead, we aim to extract the intersection of the $\mathrm{KH}$ on 2-D space of any two of the delays". This would serve a simpler visualization of the problem.

Next, we present some key definitions of time-delayed systems starting with the complete set of the imaginary eigenvalues $\boldsymbol{\Omega}$ of (2) for all possible variations of the delay vector $\boldsymbol{\tau} \in \mathfrak{R}^{p+}$

$$
\begin{aligned}
\boldsymbol{\Omega} & =\left\{\omega \mid g(s=\omega i, \boldsymbol{\tau})=0, \quad \boldsymbol{\tau} \in \mathfrak{R}^{p+}, \quad \omega \in \mathfrak{R}^{+}\right\} \\
& =\left\{\omega \mid\langle\boldsymbol{\tau}, \omega\rangle, \quad \boldsymbol{\tau} \in \mathfrak{R}^{p+}, \quad \omega \in \mathfrak{R}^{+}\right\}
\end{aligned}
$$

where $\langle\boldsymbol{\tau}, \omega\rangle$ notation implies that for a specific combination of delays as $\boldsymbol{\tau} \in \mathfrak{R}^{p+}$, there exists an imaginary root, $\omega \in \mathfrak{R}^{+}$of (2). From the set $\boldsymbol{\Omega}$ two important definitions arise.

Definition 1: Kernel hypersurfaces $(K H) \wp_{0}$ : The loci of all the points with $\langle\boldsymbol{\tau}, \omega \in \mathbf{\Omega}\rangle$ correspondence and satisfy the constraint $0<\tau_{j} \omega<2 \pi, \quad j=1,2, \ldots, p$ are called the kernel hypersurfaces. Notice that the points on these hypersurfaces have the smallest delay compositions which correspond to the same imaginary root $\omega$.
Definition 2: Offspring hypersurfaces $(\mathrm{OH}) \wp:$ The hypersurfaces obtained from $\wp_{0}$ by the following pointwise nonlinear transformation

$\left\langle\left\{\tau_{1} \pm \frac{2 \pi}{\omega} j_{1}, \tau_{2} \pm \frac{2 \pi}{\omega} j_{2}, \ldots, \tau_{p} \pm \frac{2 \pi}{\omega} j_{p}\right\}, \omega\right\rangle, \quad j_{k}=1,2, \ldots, \quad k=1,2, \ldots, p$

are called the offspring hypersurfaces. The union of $\mathrm{KH}$ and $\mathrm{OH}$ is defined as $\mathrm{KOH}$, i.e., $\bar{\wp}=\wp_{0} \cup \wp$.

Definition 3: Root tendency (RT): The root tendency is the transition direction of the imaginary root $\omega i$, when only one of the delays, say $\tau_{j}$, increases by $\varepsilon, 0<\varepsilon<<1$, while all the others remain fixed.

$$
R T_{s=\omega i}^{\tau_{j}}=\operatorname{sgn}\left[\operatorname{Re}\left(\left.\frac{\partial s}{\partial \tau_{j}}\right|_{s=\omega i}\right)\right]
$$

Clearly $R T=+1$ indicates destabilizing crossing at the imaginary axis and $R T=-1$ implies stabilizing crossing..

Exclusion 1: We limit the analysis in this paper to the most general time-delayed systems for which the imaginary spectra $\boldsymbol{\Omega}$ entails only simple eigenvalues for all delay compositions $\boldsymbol{\tau} \in \mathfrak{R}^{p+}$. In extremely rare and degenerate cases $\boldsymbol{\Omega}$ may contain multiple, identical imaginary roots for some particular delay values where the RT in (5) becomes cumbersome to determine. Such degeneracies are kept outside the scope of the paper. If they arise, however, we use a simple deployment of numerical tools (such as QPmR [40], DDE-BIFTOOL [8]) which can reveal the local root transition features near the particular point $\boldsymbol{\tau} \in \mathfrak{R}^{p+}$. Interested readers are also referred to an elaborate treatment on such degeneracies, but for much simpler single delay cases [4] and [5]. These in-depth investigations also resort to DDE-BIFTOOL to cross-validate their RT determinations.

\section{A. Half-angle Tangent Substitution}

The aim is to determine $\boldsymbol{\Omega}$ on an arbitrarily-selected 2delay cross-section of $\mathrm{KH}$, take for instance $\left(\tau_{1}, \tau_{2}\right)$ without loss of generality, while all the remaining delays $\tau_{3}, \tau_{4}, \ldots, \tau_{p}$ are fixed. For a root $s=\omega i$ we recite the Euler's formula for the transcendental terms in (2)

$$
e^{-\tau_{k} \omega i}=\cos \left(v_{k}\right)-i \sin \left(v_{k}\right), \quad v_{k}=\tau_{k} \omega, \quad k=1,2
$$

and express them in terms of a single parameter, the halfangle tangent:

$$
\cos \left(v_{k}\right)=\frac{1-z_{k}^{2}}{1+z_{k}^{2}}, \quad \sin \left(v_{k}\right)=\frac{2 z_{k}}{1+z_{k}^{2}}, \quad z_{k}=\tan \left(\frac{v_{k}}{2}\right), \quad k=1,2
$$

Notice that the inverse relation from $z_{k}$ to $\tau_{k}$ is multivalued as

$$
\tau_{k}=\frac{2 \tan ^{-1}\left(z_{k}\right)}{\omega}+\frac{2 r \pi}{\omega}, \quad 0 \leq \tan ^{-1}(.)<\pi, \quad k=1,2, \quad r=0,1,2, \ldots
$$

Substituting (7) into (2) for a given $\omega$, one obtains a new characteristic equation $\quad \widetilde{g}\left(\omega i, \mathbf{z}, e^{-\tau_{3} \omega i}, \ldots, e^{-\tau_{p} \omega i}\right)=0$ where $\mathbf{z}=\left(z_{1}, z_{2}\right) \in \mathfrak{R}^{2}$. After this domain transformation from $\boldsymbol{\tau}$ to $\left\{\mathbf{z}, \tau_{3}, \tau_{4}, \ldots\right\}$ and eliminating the $1+z_{k}^{2}$ terms in the 
denominators one obtains a modified quasi-polynomial characteristic equation as

$\bar{g}\left(\omega i, \mathbf{z}, e^{-\tau_{3} \omega i}, \ldots, e^{-\tau_{p} \omega i}\right)=\widetilde{g}\left(\omega i, \mathbf{z}, e^{-\tau_{3} \omega i}, \ldots, e^{-\tau_{p} \omega i}\right) \prod_{k=1}^{2}\left(1+z_{k}^{2}\right)^{c_{k}}=0$

where $c_{k}=\operatorname{rank}\left(\mathbf{B}_{k}\right) \leq n$ that is the commensurate degree of $\tau_{k}$.

\section{Main results}

The main motivation of the paper is to create the 2-D cross-section of the $\mathrm{KH}$ in $\left(\tau_{1}, \tau_{2}\right)$ space for a $p$-delay system when all the remaining delays $\tau_{3}, \tau_{4}, \ldots, \tau_{p}$ are arbitrarily fixed, without loss of generality. Notice that from $\mathrm{KH}$ one can create the complete $\mathrm{KOH}$ using (4). Suppressing the fixed delays in the arguments of equation (9) and for imaginary roots $s=\omega i$, one obtains

$$
\hat{g}\left(\omega i, z_{1}, z_{2}\right)=\left.\bar{g}\left(s, \mathbf{z}, e^{-\tau_{3} s}, \ldots, e^{-\tau_{p} s}\right)\right|_{s=\omega i}=0
$$

Let us denote the complete set of $\omega^{\prime} s$ satisfying (10) by $\overline{\boldsymbol{\Omega}}, \overline{\boldsymbol{\Omega}} \subset \boldsymbol{\Omega}$. To determine the entire frequency set $\overline{\boldsymbol{\Omega}}$, we deploy the Dixon resultant theory ([3], [7], [24]) for the first time, to the best of our knowledge.

Any solution $\omega \in \mathfrak{R}^{+}$of (10) has to make both real and imaginary parts of this equation vanish

$$
\begin{aligned}
& R\left(\omega, z_{1}, z_{2}\right)=\operatorname{Re}\left[\hat{g}\left(\omega i, z_{1}, z_{2}\right)\right]=\sum_{j=0}^{2 c_{2}} a_{j}\left(\omega, z_{1}\right) z_{2}^{j}=0 \\
& I\left(\omega, z_{1}, z_{2}\right)=\operatorname{Im}\left[\hat{g}\left(\omega i, z_{1}, z_{2}\right)\right]=\sum_{j=0}^{2 c_{2}} b_{j}\left(\omega, z_{1}\right) z_{2}^{j}=0
\end{aligned}
$$

These equations represent two different hypersurfaces in $\left(\omega, z_{1}, z_{2}\right) \in \mathfrak{R}^{3}$ space, say $S_{R}$ and $S_{I}$, respectively. Their intersections form the common solution of (11) and (12), which indeed reveal the entirety of $\mathrm{KOH}$

$$
\mathrm{KOH} \rightarrow S_{C}\left(\omega, z_{1}, z_{2}\right)=S_{R}\left(\omega, z_{1}, z_{2}\right) \cap S_{I}\left(\omega, z_{1}, z_{2}\right)
$$

We look at these hypersurfaces more rigorously next

Theorem 1: The KOH which are represented by (13), if they exist, form continuous hypersurfaces in $\left(z_{1}, z_{2}, \omega\right) \in \mathfrak{R}^{3}$ domain.

Proof: If there is no intersection hypercurve satisfying (11) and (12) concurrently, the system represented by (2) displays no imaginary spectra for $\left(\tau_{1}, \tau_{2}\right) \in \mathfrak{R}^{2+}$ while $\left(\tau_{3}, \tau_{4}, \ldots, \tau_{p}\right)$ are fixed. In other words, the stability of this system is independent from $\left(\tau_{1}, \tau_{2}\right)$ delays, for the selected fixed values of the remaining $p-2$ delays. We will call this "conditional delay-independent stability" as another selection of $\left(\tau_{3}, \tau_{4}, \ldots, \tau_{p}\right)$ may reverse this feature.

If there is one intersection hypercurve, we need to prove its continuity. The proof is by contradiction. If there is such a hypercurve, $S_{C}$, its projection on the parameter space of $\left(z_{1}, z_{2}\right)$ cannot be a finite curve segment $A B$ as shown in Fig. 1 [10]. Otherwise it would contradict with D-subdivision rule [25] and the root continuity rule ([18], [20]). One can move on a continuous parametric path $\left(C_{C}\right.$ in Fig. 1) and connect two points $C_{C 1}$ and $C_{C 2}$ where the number of unstable roots are different (say $N U_{1}, N U_{2}$ ) without passing through a marginal stability point on $A B$.
This is not allowed. As a consequence, the projection of $S_{C}$ on the $\left(z_{1}, z_{2}\right)$ space, i.e., the curve segment $A B$, either forms a closed loop with curve $C_{1}$ or extends to infinity as curve $C_{2}$ does in Fig. 1. Continuity condition is proven.

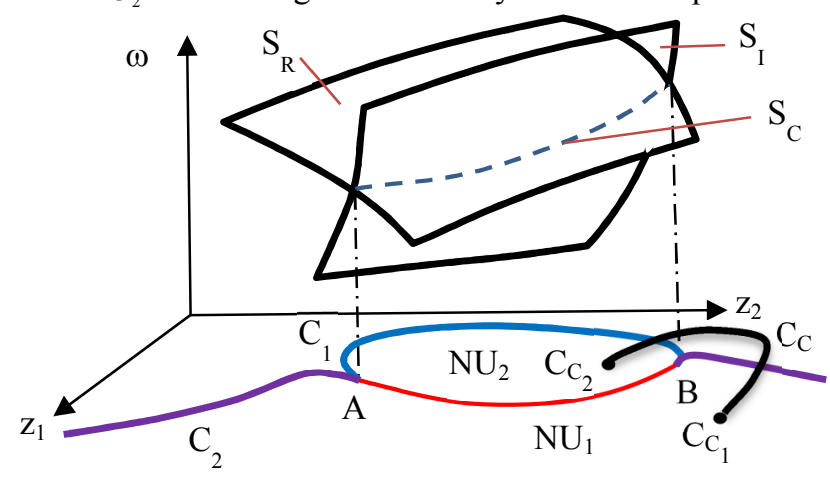

Fig. 1. $S_{C}$ as intersection of $S_{R}$ and $S_{I}$ and its projection on $\left(\mathrm{z}_{1}, \mathrm{z}_{2}\right)$ domain

With the proof of the continuity of the $\mathrm{KOH}$ in $\left(z_{1}, z_{2}, \omega\right) \in \mathfrak{R}^{3}$ domain, we next deploy the Dixon resultant methodology for the common root $z_{2}$ of the two equations (11) and (12).

\section{A. Dixon Resultant Concept and Discriminant for Pol- ynomials}

Dixon resultant is a concept on the necessary and sufficient conditions for a set of polynomial equations to possess common solutions ([3], [7]). It is one of many peer methodologies, such as Sylvester [39], Macaulay [26] and Sparse [2] resultant formulations. The advantage of the Dixon resultant procedure is its computational efficiency over the others [23]. Only a brief overview of the Dixon resultant theory from [24] is presented here for clarity.

We rewrite (11) and (12) as polynomials of $z_{2}$ with coefficients parameterized in $\omega$ and $z_{1}$

$$
\begin{gathered}
p_{1,\left[\omega, z_{1}\right]}\left(z_{2}\right) \equiv \operatorname{Re}\left[\hat{g}\left(\omega i, z_{1}, z_{2}\right)\right]=0 \\
p_{2,\left[\omega, z_{1}\right]}\left(z_{2}\right) \equiv \operatorname{Im}\left[\hat{g}\left(\omega i, z_{1}, z_{2}\right)\right]=0
\end{gathered}
$$

and seek the necessary and sufficient conditions for a common $z_{2}$ solution to exist. The method starts with the following Dixon polynomial in $z_{2}$

$$
\delta\left(z_{2}, \alpha\right)=\frac{1}{\left(z_{2}-\alpha\right)}\left|\begin{array}{ll}
p_{1,\left[\omega, z_{1}\right]}\left(z_{2}\right) & p_{2,\left[\omega, z_{1}\right]}\left(z_{2}\right) \\
p_{1,\left[\omega, z_{1}\right]}(\alpha) & p_{2,\left[\omega, z_{1}\right]}(\alpha)
\end{array}\right|
$$

where $p_{i,\left[\omega, z_{1}\right]}(\alpha), i=1,2$ stands for replacing $z_{2}$ by $\alpha$, a dummy variable. Equation (16) is symmetric with respect to $\alpha$ and $z_{2}$, i.e., $\delta\left(\alpha, z_{2}\right)=\delta\left(z_{2}, \alpha\right)$. Furthermore $\delta$ is of degree $\quad d_{\max }-1 \quad$ in $\quad \alpha \quad$ where $d_{\text {max }}=\max \left[\operatorname{deg}\left(p_{1,\left[\omega, z_{1}\right]}, z_{2}\right), \operatorname{deg}\left(p_{2,\left[\omega, z_{1}\right]}, z_{2}\right)\right]$ and the notation $\operatorname{deg}\left(p, z_{2}\right)$ is used to represent the maximum degree of $z_{2}$ in the polynomial $p$. Since each common zero of $p_{1,\left[\omega, z_{1}\right]}\left(z_{2}\right)$ and $p_{2,\left[\omega, z_{1}\right]}\left(z_{2}\right)$ is a zero of $\delta\left(z_{2}, \alpha\right)$ regardless of $\alpha$ values, the coefficients of all powers of $\alpha$ 
in $\delta\left(z_{2}, \alpha\right)$ must be zero at this common zero. This yields $d_{\max }$ equations corresponding to the coefficients of $d$ $\left(i=0,1, \ldots, d_{\max }-1\right)$ which are all polynomials in $z_{2}$. The corresponding coefficient matrix $\mathbf{F}\left(\omega, z_{1}\right) \in \mathfrak{R}^{d_{\max } \times d_{\max }}$ of these $d_{\max }$ equations is known as the Dixon matrix

$$
\mathbf{F}\left(\omega, z_{1}\right)\left(\begin{array}{c}
1 \\
z_{2} \\
\vdots \\
z_{2}^{d_{\max }-1}
\end{array}\right)=\left(\begin{array}{c}
0 \\
0 \\
\vdots \\
0
\end{array}\right)
$$

For a solution of (17) to exist, $\mathbf{F}\left(\omega, z_{1}\right)$ has to be singular

$$
\operatorname{det}\left[\mathbf{F}\left(\omega, z_{1}\right)\right]=0
$$

which provides the necessary and sufficient condition we are seeking. The determinant in (18) is known to be the Dixon Resultant [24]. We denote it as $R z_{2}\left(\omega, z_{1}\right)$ $=\operatorname{det}\left[\mathbf{F}\left(\omega, z_{1}\right)\right]$. We wish to formalize the above development as a theorem.

Theorem 2 [3]: The necessary and sufficient condition for two polynomials $p_{1,\left[\omega, z_{1}\right]}\left(z_{2}\right)$ and $p_{2,\left[\omega, z_{1}\right]}\left(z_{2}\right)$ to have a nontrivial common zero is that the corresponding Dixon matrix $\mathbf{F}\left(\omega, z_{1}\right)$ is singular, i.e.

$$
R z_{2}\left(\omega, z_{1}\right)=\operatorname{det}\left[\mathbf{F}\left(\omega, z_{1}\right)\right]=0
$$

In light of the constructive suggestions of an anonymous reviewer we include the following preclusion clause. This has to do with the differentiability of the $\mathrm{KOH}$.

Assumption 1: For the transcendental equations in (11) and (12) the differentiability with respect to the arguments $\left(\omega, z_{1}, z_{2}\right)$ is assumed to hold at all points. Rigorous assessment of the extremely rare conditions for this assumption not to be valid in multiple delay systems is a very complicated problem, as declared in [5], and thus kept outside the discussions here.

It is obvious that the upper and lower bounds of $\omega$ is the crucial information for $\mathrm{KOH}$. If they were known one could sweep $\omega$ in this interval and obtain the corresponding $z_{1} \in \mathfrak{R}$ values from (19). With these $\omega$ and $z_{1} \in \Re$, the companion $z_{2} \in \mathfrak{R}$ values result from (11) or (12), and the delays $\left(\tau_{1}, \tau_{2}\right)$ from (8). The main motivation of the paper originates from the inquiry of the upper and lower bounds of $\omega$. Having established the continuity of $\omega$ with respect to $z_{1}$ (or $z_{2}$ ) in the previous section, we explore the extremum condition of

$$
d \omega / d z_{1}=0\left(\text { or } d \omega / d z_{2}=0\right)
$$

in the following segments.

Observation 1: We are seeking the conditions for common roots of $R z_{2}\left(\omega, z_{1}\right)=0$ (19) and $d \omega / d z_{1}=0$ (20) to exist. We also notice that the variational form of (19) along any $\mathrm{KOH}$ leads to

$$
d R z_{2}=\frac{\partial R z_{2}}{\partial \omega} d \omega+\frac{\partial R z_{2}}{\partial z_{1}} d z_{1}=0
$$

$$
\text { or } \quad \frac{d \omega}{d z_{1}}=-\frac{\partial R z_{2}}{\partial z_{1}} / \frac{\partial R z_{2}}{\partial \omega}
$$

Due to (20) last equation enforces

$$
\partial R z_{2} / \partial z_{1}=0
$$

Definition $4[15]$ : Discriminant of $R z_{2}\left(\omega, z_{1}\right):$ The discriminant of $R z_{2}\left(\omega, z_{1}\right)$ with respect to $z_{1}$, denoted by $D z_{1}(\omega)$, is the resultant formed using the two polynomials $R z_{2}\left(\omega, z_{1}\right)$ and $\partial R z_{2}\left(\omega, z_{1}\right) / \partial z_{1}$ by eliminating $z_{1}$.

This operation, in fact, is another Dixon procedure but this time in order to guarantee the common $z_{1}$ roots between (19) and (23). Notice that the polynomial $D z_{1}(\omega)$ is a function of one argument only $(\omega)$, and by solving its real roots one can find the candidate $\omega^{\prime} s$ for the upper and lower bounds of $\omega$.

\section{B. Main Theorem, the Determination of the Bounds of the Imaginary Spectra}

With the knowledge of Definition 4 and Theorem 2, we present the main theorem that reveals the exact upper and lower bounds of $\overline{\boldsymbol{\Omega}}$ for the case when the frequencies of the imaginary spectra are strictly positive, i.e., the lower bound is not zero.

Theorem 3: The upper and lower bounds of $\overline{\mathbf{\Omega}}$ are the maximum and minimum positive real roots of the discriminant of the resultant $R z_{2}$, i.e., $D z_{1}(\omega)=0$.

Proof: In accordance with Theorem 2, the resultant should vanish, $R z_{2}\left(\omega, z_{1}\right)=0$ (19). According to the earlier Observation $1 \partial R z_{2} / \partial z_{1}=0$ (23) condition must also hold. We then recognize if these two equations held concurrently, (11) and (12) would have a nontrivial common zero in $\omega$ which will be a candidate for an extremum. These arguments enforce the discriminant of the resultant $R z_{2}$, i.e., $D z_{1}(\omega)$ to vanish .QED.

$D z_{1}(\omega)$ is a function in $\omega$ only, and its real zeros can be determined. We denote all positive roots of this function as $\hat{\omega}_{1}<\hat{\omega}_{2}<\ldots<\hat{\omega}_{l-1}<\hat{\omega}_{l}, l \in Z^{+}$a positive integer. To calculate the upper bound of $\overline{\boldsymbol{\Omega}}$, we follow the steps below with the initial condition $i=l$ (i.e., the largest common frequency):

(a) Solve $R z_{2}\left(\hat{\omega}_{i}, z_{1}\right)=0$ for $z_{1} \in \mathfrak{R}$. If no real solution exists, reduce $i$ by 1 and repeat this step.

(b) For each $\left(\hat{\omega}_{i}, z_{1}\right) \in \mathfrak{R}^{2}$ composition obtained in (a), solve equation (11) or (12) for $z_{2} \in \mathfrak{R}$. If there exists no common $z_{2} \in \mathfrak{R}$ root for (11) and (12), then reduce $i$ by 1 and return to (a). If such a $z_{2} \in \mathfrak{R}$ root exists, declare $\hat{\omega}_{i}$ as the upper bound of $\overline{\mathbf{\Omega}}$ and denote it as $\bar{\omega}$.

For the lower bound $\underline{\omega}$ repeat the same steps (a-b) above except starting from $i=1$ and increasing it by 1 until 
the first $\left(z_{1}, z_{2}\right) \in \mathfrak{R}^{2}$ are obtained. Label the corresponding $\hat{\omega}_{i}$ as $\underline{\omega}$. Departing from these upper and lower bounds of $\overline{\mathbf{\Omega}}$, the following numerical procedures are performed to create the target 2-D cross-section of the $p$-D KH.

(i) Sweep $\omega$ within the obtained bounds using a desirably small step size and solve $R z_{2}\left(\omega, z_{1}\right)=0$ for $z_{1} \in \mathfrak{R}$.

(ii) For each $\left(\omega, z_{1}\right) \in \mathfrak{R}^{2}$ composition in (i), check if $z_{2} \in \mathfrak{R}$ exists to satisfy (11) and (12). If so, go to step (iii). Otherwise, return to (i) and continue sweeping to the next $\omega$ value.

(iii) For the obtained $\left(\omega, z_{1}, z_{2}\right) \in \mathfrak{R}^{3}$ correspondences, determine the kernel delay composition $\left(\tau_{1}, \tau_{2}\right) \in \mathfrak{R}^{2+}$ as per (8) and the definition of $\mathrm{KH}$.

This procedure results in the 2-D intersection of the $p$-D $\mathrm{KH}$ in $\left(\tau_{1}, \tau_{2}\right) \in \mathfrak{R}^{2+}$ space. As explained earlier $\mathrm{KH}$ is all one needs to obtain the complete $\mathrm{OH}$ and the complete 2-D intersection of the stability map in $\left(\tau_{1}, \tau_{2}\right) \in \mathfrak{R}^{2+}$ space.

Remark 1: Dixon resultant is computationally more efficient over the peer resultant formulations. Specifically, the preference of the Dixon resultant over the well-known Sylvester resultant is due to its faster computation speed for various practical cases [24]. The size of the resultant matrix is critical in determining the efficiency of any of these methods [23]. Dixon resultant evaluates the determinant of a $\max (m, n) \times \max (m, n)$-dimensional Dixon matrix $\mathbf{F}$, where $m=\operatorname{deg}\left(p_{1,\left[\omega, z_{1}\right]}, z_{2}\right) \quad$ and $n=\operatorname{deg}\left(p_{2,\left[\omega, z_{1}\right]}, z_{2}\right)$. This matrix is much smaller than the $(m+n) \times(m+n) \quad$-dimensional Sylvester matrix. Appendix A contains a simple example to illustrate this comparison. We will also present the computational advantage of Dixon resultant over Sylvester resultant using an example in the next section.

\section{Example case study}

In order to demonstrate the capabilities of the proposed method, we take a system with $n=2, p=5$ :

$$
\begin{aligned}
& \mathbf{A}=\left[\begin{array}{cc}
0 & 3 \\
-24 & -9
\end{array}\right], \mathbf{B}_{1}=\left[\begin{array}{ll}
-1 & 0.5 \\
-2 & -3
\end{array}\right], \mathbf{B}_{2}=\left[\begin{array}{cc}
-2 & 0.1 \\
1 & -1
\end{array}\right], \\
& \mathbf{B}_{3}=\left[\begin{array}{cc}
1 & 3 \\
-5 & 0.2
\end{array}\right], \quad \mathbf{B}_{4}=\left[\begin{array}{ll}
2 & 0.5 \\
1 & -1
\end{array}\right], \quad \mathbf{B}_{5}=\left[\begin{array}{cc}
3 & 0 \\
-2 & 2
\end{array}\right]
\end{aligned}
$$

Notice that $\mathbf{B}_{i}$ matrices all have the rank 2, i.e., $c_{i}=2, \quad i=1,2, \ldots, 5$. Therefore characteristic equation contains some double-commensurate delay expressions, such as $e^{-2 \tau_{1} s}$

$$
\begin{aligned}
& g(s, \tau)=s^{2}+9 s+72+(4 s+27) e^{-\tau_{1} s}+(3 s+17.4) e^{-\tau \tau_{2} s}+(-1.2 s+78) e^{-\tau_{3} s}-(s \\
& +9) e^{-\tau_{4} s}-(5 s+21) e^{-\tau_{s} s}+4 e^{-2 \tau_{1} s}+1.9 e^{-2 \tau_{2} s}+15.2 e^{-2 \tau_{s} s}-2.5 e^{-2 \tau_{4} s}+6 e^{-2 \tau_{s} s} \\
& +6.7 e^{-\left(\tau_{1}+\tau_{2}\right) s}+5.3 e^{-\left(\tau_{1}+\tau_{3}\right) s}-4.5 e^{-\left(\tau_{1}+\tau_{4}\right) s}-10 e^{-\left(\tau_{1}+\tau_{5}\right) s}-3.9 e^{-\left(\tau_{2}+\tau_{3}\right) s}-0.6 e^{-\left(\tau_{2}+\tau_{4}\right) s} \\
& -6.8 e^{-\left(\tau_{2}+\tau_{5}\right) s}-1.1 e^{-\left(\tau_{3}+\tau_{4}\right) s}+8.6 e^{-\left(\tau_{3}+\tau_{5}\right) s}+2 e^{-\left(\tau_{4}+\tau_{5}\right) s}=0
\end{aligned}
$$

In this equation, we arbitrarily fix $\tau_{3}=0.5, \tau_{4}=1.5$ and $\tau_{5}=1$. Notice the critical nuance that $\tau_{3}$ is selected independently and fixed, although $\tau_{4}$ and $\tau_{5}$ are commensurate delays vis-à-vis $\tau_{3}$ for numerical convenience only. So the problem is indeed in the domain of two completely independent delays $\left(\tau_{1}, \tau_{2}\right) \in \mathfrak{R}^{2+}$. We suppress the steps involving the resultant, as in (18), and discriminant as in Definition 4, due to space constraints. According to Theorem 3, for this system and the three fixed delays the exact lower and upper bounds of the set $\overline{\mathbf{\Omega}}$ is determined to be $[3.8931,13.3238]$. Next, we apply the CTCR paradigm to reveal the stability map in $\left(\tau_{1}, \tau_{2}\right)$ space as shown in Fig. 2. In Fig.2 over some distinct segments of $\mathrm{KOH}$ the complete variation of $\omega$ is also shown as insets. There are two loops forming the $\mathrm{KH}$ (one is intact and the other is split in four parts to protect $\tau>0$ condition). It is easy to observe the proven continuity and assumed differentiability $d \omega / d \tau_{1}, d \omega / d \tau_{2}$ on the insets. The deployment of CTCR starts with the determination of the stability at point $\tau_{1}=0, \tau_{2}=0, \tau_{3}=0.5, \tau_{4}=1.5$ and $\tau_{5}=1$. For this we could simply use some numerical methods, for instance the Quasi-Polynomial mappingbased Root-finder (QPmR) algorithm [40]. It declares "stable" behavior at $\left(\tau_{1}, \tau_{2}\right)=(0,0)$ point and we progress further examining the NU variations at partitions of the delay space (which are created by $\mathrm{KOH}$ ) as displayed sparsely in Fig. 2, shaded regions are the stable regions with $\mathrm{NU}=0$.

Remark 2: For the calculation of $D z_{1}(\omega)$, i.e., the discriminant of $R z_{2}$, we utilized two polynomials, i.e., $R z_{2}$ and $d R z_{2} / d z_{1}$. In the example above, the highest order of $z_{1}$ in $R z_{2}$ is $z_{1}^{8}$. Hence, $d R z_{2} / d z_{1}$ is a $7^{\text {th }}$ degree polynomial in $z_{1}$. According to Remark 1, the Dixon resultant evaluates the determinant of an $8 \times 8$ matrix while the Sylvester resultant calculates the determinant of a $15 \times 15$ matrix. The Dixon resultant requires 380 seconds with a storage consumption of 962 Megabytes on a standard desktop with $2.9 \mathrm{GHz} \mathrm{CPU}$ and 3 GB RAM. On the same computer, the computation of the symbolic evaluation of Sylvester resultant encounters memory space limitation and halts.

\section{Conclusion}

This paper introduces some new perspectives on the CTCR stability analysis of the general LTI-MTDS with 
arbitrarily large number of delays. A novel procedure is presented to create the stability partitioning kernel and offspring hypersurfaces $(\mathrm{KOH})$ exhaustively for such systems. This procedure combines the strength of the frequency sweeping technique and the Dixon resultant theory. And it declares the cross-section of the general $p$-D stability maps on the space of any of the two delays (2-D) by fixing the remainder of the delays at arbitrarily selected values. This is achieved by first extracting the bounds of the frequency of the imaginary root crossings for all possible delay compositions, and then sweeping this frequency interval.

The text provides the proof of the continuity of these crossing-frequency variations. With this information, the complete $\mathrm{KOH}$ on any 2-dimensional delay space the exact and exhaustive stability map is constructed as per the CTCR paradigm. One case study with five delays (three independent and two commensurate) is presented to show the validity and strength of the proposed procedure.

\section{References}

[1] Almodaresi, E. and Bozorg, M. (2009). Stability crossing surfaces for linear time-delay systems with three delays. International Journal of Control, 82(12), 2304-2310.

[2] Canny, J. and Emiris, I. (1993). An efficient algorithm for the sparse mixed resultant. In Applied Algebra, Proceedings of the Algebraic Algorithms and Error-correcting Codes, Springer Berlin Heidelberg, 89-104.

[3] Cayley, A. (1865). On the theory of elimination. Cambridge and Dublin Mathematical Journal, III, 210-270.

[4] Chen, J., Fu, P., Niculescu, S. -I. and Guan, Z. (2010). An eigenvalue perturbation approach to stability analysis, Part I: eigenvalue series of matrix operators. SIAM J. Control Optim, 48(8), 5564-5582.

[5] Chen, J., Fu, P., Niculescu, S. -I. and Guan, Z. (2010). An eigenvalue perturbation approach to stability analysis, Part II: when will zeros of time-delay systems cross imaginary axis? SIAM J. Control Optim, 48(8), 5583-5605.

[6] Chen, J. and Latchman, H. A. (1995). Frequency sweeping tests for asymptotic stability independent of delay. IEEE Transactions on Automatic Control, 40(9), 1640-1645.

[7] Dixon, A. L. (1908). The eliminant of three quantics in two independent variables. In Proceedings of the London Mathematical Society, 6, 468-478.

[8] Engelborghs, K., Luzyanina, T. and Roose, D. (2002). Numerical bifurcation analysis of delay differential equations using DDE-BIFTOOL, ACM Trans. Math. Software, 28, 121.

[9] Ergenc, A. F., Olgac, N., and Fazelinia, H. (2007). Extended kronecker summation for cluster treatment of LTI systems with multiple delays. SIAM Journal on Control and Optimization, 46(1), 143-155.

[10] Fazelinia, H., Sipahi, R., and Olgac, N. (2007). Stability robustness analysis of multiple time-delayed systems using 'Building Block' concept. IEEE Transactions on Automatic Control, 52(5), 799-810.

[11] Gao, Q., Kammer, A.S., Zallugoglu, U., and Olgac, N. (2015). Combination of sign inverting and delay scheduling control concepts for multiple-delay dynamics. Systems \& Control Letters, 77, 55-62.

[12] Gao, Q., Kammer, A.S., Zallugoglu, U., and Olgac, N. (2015). Critical effects of the polarity change in delayed states within an LTI Dynamics with multiple delays. IEEE Transactions on Automatic Control, 60(11), 3018-3022.

[13] Gao, Q. and Olgac, N. (2015). Optimal sign inverting control for time-delayed systems, a concept study with experiments. International Journal of Control, 88(1), 113-122.
[14] Gao, Q., Zalluhoglu, U., and Olgac, N. (2014) Investigation of local stability transitions in the spectral delay space and delay space. ASME Journal of Dynamic Systems, Measurement and Control, 136(5), 77-85.

[15] Gelfand, I. M., Kapranov, M. M., and Zelevinsky, A. V. (1994). Discriminants, Resultants, and Multidimensional Determinants. Boston, MA: Birkhauser.

[16] Gu, K. and Naghnaeian, M. (2011). Stability crossing set for systems with three delays. IEEE Transactions on Automatic Control, 56(1), 11-26.

[17] Gu, K., Niculescu, S. -I., and Chen, J. (2005). On stability crossing curves for general systems with two delays. Journal of Mathematical Analysis and Applications, 311(1), 231253.

[18] Hale, J. K. (1977). Theory of functional differential equations. New York: Springer- Verlag.

[19] Hale, J. K., Infante, E. F., and Tsen, F. S. P. (1985). Stability in linear delay equations. Journal of Mathematical Analysis and Applications, 105(2), 533-555.

[20] Hale, J. K. and Lunel, S. M. V. (1993). An introduction to functional differential equations. New York: SpringerVerlag.

[21] Hartmann, E. (2003). Geometry and algorithms for computer aided design, Darmstadt University, page 55.

[22] Jarlebring, E. (2009). Critical delays and polynomial eigenvalue problems. Journal of Computational and Applied Mathematics, 224(1), 296-306.

[23] Kapur, D. and Saxena, T. (1995). Comparison of various multivariate resultant formulations. In Proceedings of the International Symposium on Symbolic and Algebraic Computation, ACM, 187-194.

[24] Kapur, D., Saxena, T. and Yang, L. (1994). Algebraic and geometric reasoning using Dixon resultants. In Proceedings of the International Symposium on Symbolic and Algebraic Computation, ACM, 99-107.

[25] Kolmanovski, V. B. and Nosov, V. R. (1986). Stability of functional differential Equations. London, U.K.: Academic.

[26] Macaulay, F. S. (1916). The algebraic theory of modular systems. Cambridge Tracts in Mathematics and Mathematical Physics, No.19.

[27] Michiels, W. and Niculescu, S. -I. (2007). Stability and stabilization of time-delay systems: An eigenvalue-based approach. In Advances in design and control: Vol. 12. Philadelphia: SIAM.

[28] Niculescu, S. -I. (1998). Stability and hyperbolicity of linear systems with delayed state: a matrix-pencil approach. Journal of Mathematical Control and Information, 15, 331-347.

[29] Olgac, N. and Sipahi, R. (2002). An exact method for the stability analysis of time delayed LTI systems. IEEE Transactions on Automatic Control, 47(5), 793-797.

[30] Packard, A. and Doyle, J. C. (1993). The complex structured singular value. Automatica, 29(1), 71-109.

[31] Rekasius, Z. V. (1980). A stability test for systems with delays. In Proceedings of the Joint Automatic Control Conference. San Francisco, CA, pp. TP9-A.

[32] Sipahi, R. and Delice, I. I. (2009). Extraction of 3D stability switching hypersurfaces of a time delay system with multiple fixed delays. Automatica, 45(6), 1449-1454.

[33] Sipahi, R. and Delice, I. I. (2011). Advanced clustering with frequency sweeping methodology for the stability analysis of multiple time-delay systems. IEEE Transactions on Automatic Control, 56(2), 467-472.

[34] Sipahi, R. and Olgac, N. (2006a). A unique methodology for the stability robustness of multiple time delay systems. Systems and Control Letters, 55(10), 819-825.

[35] Sipahi, R. and Olgac, N. (2006b). An improved procedure in detecting stability robustness of systems with uncertain delay. IEEE Transactions on Automatic Control, 51(7), 1164 1165.

[36] Sipahi, R., Olgac, N., and Breda, D. (2010). A stability study on first order neutral systems with three rationally independent time delays. International Journal of Systems Science, 41(12), 1445-1455. 
[37] Spivak, M. (2006). Calculus, Cambridge University Press, $382-383$.

[38] Toker, O. and Ozbay, H. (1996). Complexity issues in robust stability of linear delay-differential systems. Mathematics of Control, Signals and Systems, 9(4), 386-400.

[39] Trott, M. (2006). The mathematica guidebook for symbolics. New York: Springer-Verlag, page 28.

[40] Vyhlidal, T., and Zitek, P. (2009). Mapping based algorithm for large scale computation of quasi polynomial zeros. IEEE Transactions on Automatic Control, 54(1), 171-177.

\section{Appendix A. Comparative example between the Dixon and Sylvester resultant matrices}

Consider the following two polynomials

$$
\begin{array}{ll}
p_{1}(x)=a_{0}+a_{1} x, & a_{1} \neq 0 \\
p_{2}(x)=b_{0}+b_{1} x+b_{2} x^{2}, & b_{2} \neq 0
\end{array}
$$

Dixon matrix: We denote $m \equiv \operatorname{deg}\left(p_{1}, x\right)=1$, $n \equiv \operatorname{deg}\left(p_{2}, x\right)=2$ where $\operatorname{deg}\left(p_{1}, x\right)$ is the maximum degree of $x$ in $p_{1}$. According to (16), the Dixon polynomial $\delta$ is obtained as

$$
\begin{aligned}
\delta(x, \alpha)= & \frac{1}{(x-\alpha)}\left|\begin{array}{ll}
p_{1}(x) & p_{2}(x) \\
p_{1}(\alpha) & p_{2}(\alpha)
\end{array}\right|=\frac{1}{(x-\alpha)}\left|\begin{array}{ll}
a_{0}+a_{1} x & b_{0}+b_{1} x+b_{2} x^{2} \\
a_{0}+a_{1} \alpha & b_{0}+b_{1} \alpha+b_{2} \alpha^{2}
\end{array}\right| \\
& =\left(-a_{1} b_{2} x-a_{0} b_{2}\right) \alpha+\left(-a_{0} b_{1}+a_{1} b_{0}-a_{0} b_{2} x\right)
\end{aligned}
$$

Note that $d_{\max }=\max (m, n)=2$ and $\delta$ is of degree $d_{\max }-1=1$ in $\alpha$. Furthermore it is symmetric in $\alpha$ and $x$ (i.e., $\delta$ remains invariant when $\alpha$ and $x$ are swapped). Notice that each common zero of $p_{1}(x)$ and $p_{2}(x)$ is a zero of $\delta(x, \alpha)$ independently from the $\alpha$ values, therefore the coefficients of powers of $\alpha$ in $\delta(x, \alpha)$ must be zero. This results in $d_{\max }=2$ equations corresponding to the coefficients (which are polynomials in $x$ ) of $\alpha^{i}(i=0,1)$ :

$$
\left\{\begin{array}{l}
-a_{0} b_{1}+a_{1} b_{0}-a_{0} b_{2} x=0 \\
-a_{1} b_{2} x-a_{0} b_{2}=0
\end{array}\right.
$$

The coefficient matrix $\mathbf{F} \in \mathfrak{R}^{2 \times 2}$ of these $d_{\max }=2$ equations is known as the Dixon matrix.

$$
\mathbf{F}\left(\begin{array}{l}
1 \\
x
\end{array}\right) \equiv\left[\begin{array}{cc}
-a_{0} b_{1}+a_{1} b_{0} & -a_{0} b_{2} \\
-a_{0} b_{2} & -a_{1} b_{2}
\end{array}\right]\left(\begin{array}{l}
1 \\
x
\end{array}\right)=\left(\begin{array}{l}
0 \\
0
\end{array}\right)
$$

For a nontrivial solution of this equation $\mathbf{F}$ must be singular:

$$
a_{0}^{2} b_{2}-a_{0} a_{1} b_{1}+a_{1}^{2} b_{0}=0
$$

Sylvester matrix: The Sylvester matrix of $p_{1}$ and $p_{2}$ with respect to $x$ (denoted by $\mathbf{S}$ ) is formed by the coefficients of $x$ in equations (A1) as the following $(m+n) \times(m+n)=3 \times 3$ matrix

$$
\mathbf{S}=\left(\begin{array}{ccc}
a_{1} & a_{0} & 0 \\
0 & a_{1} & a_{0} \\
b_{2} & b_{1} & b_{0}
\end{array}\right)
$$

For a common solution of (A1) to exist $\mathbf{S}$ needs to be singular (i.e., its determinant known as the Sylvester resultant has to be zero)

$$
\operatorname{det}(\mathbf{S})=a_{0}^{2} b_{2}-a_{0} a_{1} b_{1}+a_{1}^{2} b_{0}
$$

which gives the identical condition as in (A5).

For this explanatory example, the Dixon matrix $\mathbf{F} \in \mathfrak{R}^{2 \times 2}$ is smaller than the Sylvester matrix $\mathbf{S} \in \mathfrak{R}^{3 \times 3}$, and computational simplicity is obvious. In general, the dimension of Dixon matrix is $\max (m, n) \times \max (m, n)$ and the Sylvester matrix is $(m+n) \times(m+n)$ which is clearly much larger. The difference can be substantial, especially when $m$ or $n$ is large.

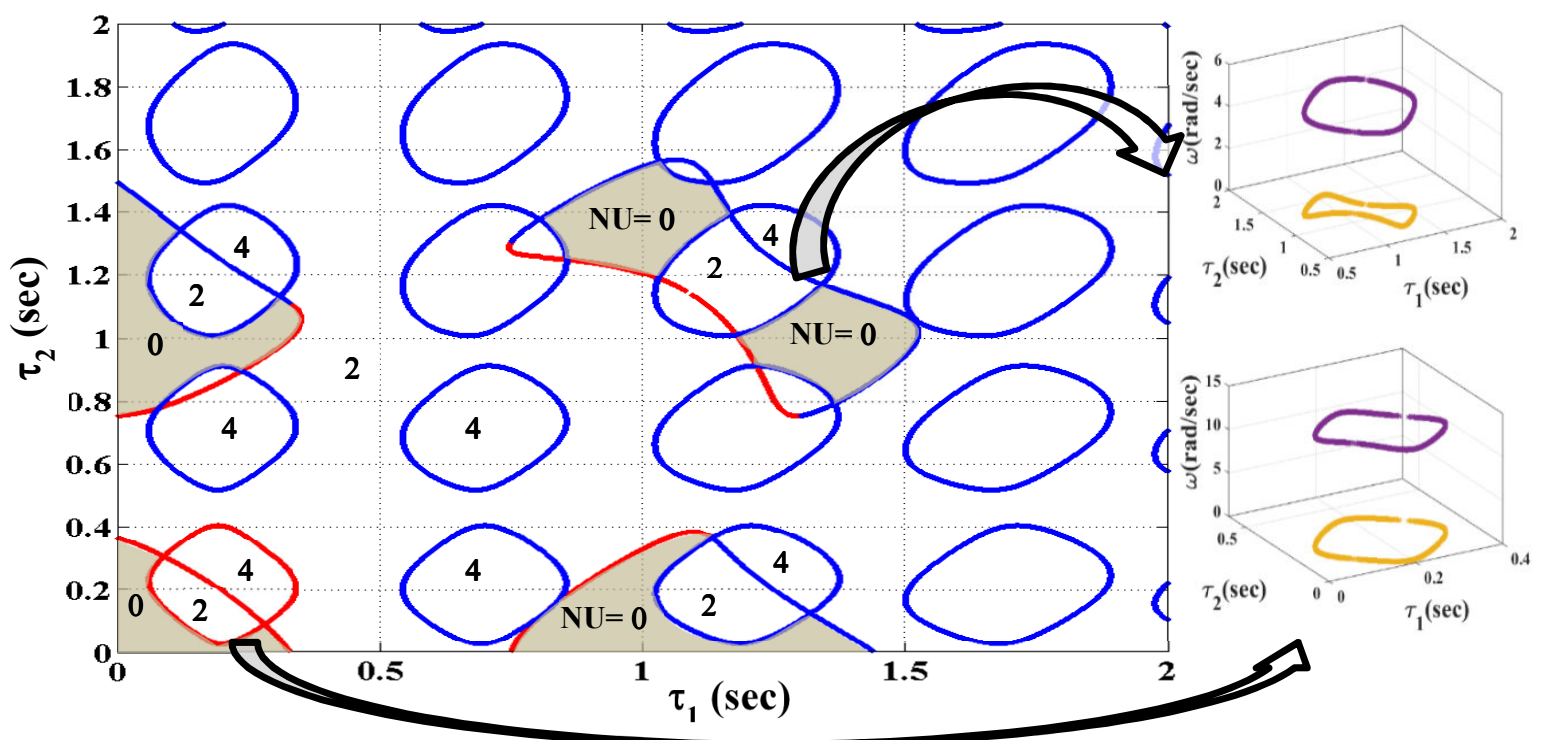

Fig. 2. Stability map of the example case (24) for $\tau_{3}=0.5 \mathrm{sec}, \tau_{4}=1.5 \mathrm{sec}, \tau_{5}=1 \mathrm{sec}$; shaded regions are stable; red and blue curves are $\mathrm{KH}$ and $\mathrm{OH}$, respectively. 